\title{
Evaluation of horn bud wound healing following cautery disbudding of preweaned dairy calves treated with aluminum-based aerosol bandage
}

\author{
K. L. Huebner, A. K. Kunkel, C. S. McConnel, R. J. Callan, R. P. Dinsmore, and L. S. Caixeta ${ }^{1}$ \\ Department of Clinical Sciences, Colorado State University, Fort Collins 80523
}

\begin{abstract}
Pain management during and following disbudding procedures has been studied extensively, though few studies have evaluated wound healing following cautery disbudding in dairy calves. The purpose of this study was to observe wound healing following cautery disbudding with or without treatment using a topical aluminum-based aerosol bandage (ALU) in preweaned dairy calves. Dairy calves were disbudded within the first 3 wk of life using a standard cautery disbudding protocol. The ALU treatment was randomly allocated to the right or left horn bud within each animal. The outcomes measured were lesion score (LS) and wound diameter (WD). The LS was evaluated on a scale of 1 to 3 , with LS $=1$ representing normal healing without a scab or exudate, $\mathrm{LS}=2$ having the presence of a scab, and LS $=3$ showing the presence of wound exudate. Lesion score and WD were evaluated on a weekly basis following dehorning for 3 wk. A total of 209 animals completed the study. No difference was observed in LS between groups during the first 2 wk postdisbudding, but the proportion of LS $=3$ on wk 3 postdisbudding was greater for the control group when compared with ALU (17 vs. 8\%, respectively). During wk 1 and 2 postdisbudding, the odds of having delayed healing, or a LS $\geq 2$, were similar for both groups. However, the odds tended to be different at wk 3 postdisbudding with control disbudding sites being 1.42 times more likely to have delayed healing than ALU. In wk 3, WD was 1 $\mathrm{mm}$ smaller in the treatment group compared with the control, and treatment decreased diameter over time compared with controls. Overall, once abnormal wound healing was observed, the likelihood of having abnormal wound healing the following week was increased. However, treatment with ALU diminished this effect on delayed healing during the follow-up period. Based on these results, the use of ALU improved wound healing following cautery disbudding of preweaned dairy calves.
\end{abstract}

Received October 21, 2016.

Accepted January 16, 2017

${ }^{1}$ Corresponding author: luciano.caixeta@colostate.edu
Key words: disbudding, dairy calf, wound healing, topical aluminum spray

\section{INTRODUCTION}

Disbudding procedures are commonly performed on dairy operations to prevent horn-related trauma due to aggressive behavior, to allow for safer cattle handling, to decrease feed bunk space requirements, and to decrease carcass bruising in dairy cattle at slaughter (Ramsay et al., 1976; Marshall, 1977). With the increasing social concern and awareness for animal welfare, disbudding of dairy calves has received increasing public attention, particularly with regard to pain mitigation (Robbins et al., 2015). Arguably, healing of postdisbudding wound sites also poses a welfare issue to calves, although no studies to date have assessed this issue (Stafford and Mellor, 2005).

Several management practices are used to remove horns in cattle and they vary based on age, breed, and degree of horn development. Primary methods for dehorning of dairy cattle young stock typically include cautery removal of the horn bud or application of caustic pastes such as sodium hydroxide. As horns grow and the bovine skull develops, surgical amputation must be employed, resulting in a more invasive surgical procedure (Aubry, 2005). Regardless of the disbudding method used, the implementation of different strategies for pain control can benefit animal welfare and production after disbudding (Stock et al., 2013).

Over the last 2 decades, changes in behavior and pain associated with different methods of disbudding and dehorning have been studied using physiologic, behavioral, and performance responses in calves (Stafford and Mellor, 2005). Commonly measured parameters to quantify the pain response include overt behavioral signs, blood cortisol levels, lying time, ADG, feed intake, heart rate, vasopressin levels, adrenaline, leukocyte counts, and pressure algometry. It has been shown across several studies that lidocaine combined with a systemic nonsteroidal anti-inflammatory drug, and when possible, sedative analgesics, results in the most effective reduction in pain response in calves (Stock et 
al., 2013). In addition, analgesic protocols have been shown to improve weight gain and milk intake of dairy calves after disbudding. Particularly, calves disbudded with no pain relief or an nonsteroidal anti-inflammatory drug alone have a slower growth rate than calves that are disbudded using local anesthesia and sedation at disbudding, although the degree of effect of analgesic protocol is influenced by operator skill level while performing the procedure and time frame of analgesic application following disbudding (Bates et al., 2015).

No studies to date have evaluated wound healing postcautery disbudding in dairy calves. A recent study evaluated wound healing by various methods of dehorning in feedlot cattle and showed differences in wound infection and healing rate across dehorning methods, with healing taking up to 4 wk (Neely et al., 2014). Within the dairy industry, wound healing following horn amputation in older calves has been shown to cause chronic pain and reductions in weight gain, in part due to the slow healing time (Loxton et al., 1982; Kihurani et al., 1989). However, unlike surgical methods, cautery disbudding creates a more superficial wound that does not penetrate the skull or enter the frontal sinus. Despite this, lesions and delayed wound healing are still commonly observed in dairy calves, with unknown consequences for long-term health and production.

The efficacy of topical treatments applied to the wound site following cautery disbudding has not been reported in the literature. Aluminum-based aerosol bandage (ALU) sprays are commonly used as a protective bandage over wounds, and the product label claims that the product works as a waterproof barrier protectant and does not damage wound tissues. The exact mechanism of action for ALU is currently unknown, and no research data have supported the effectiveness of these topical wound sprays to ameliorate wound healing. The objectives of the current study were to assess the efficacy of topical ALU on wound healing following cautery disbudding of dairy calves and to evaluate wound healing progression during a 3 -wk follow-up period.

\section{MATERIALS AND METHODS}

\section{Study Population and Study Design}

All procedures involving animals were approved by the Colorado State University Research Integrity and Compliance Review Office as Veterinary Clinical Study (protocol 2015-033).

Holstein heifers from 3 commercial dairy farms in Colorado were enrolled in the study between 1 and 3 wk of age from August to October 2015. All enrolled calves were healthy and housed in calf hutches bedded on outdoor dirt, with indoor shavings or straw.

Calves were disbudded by study personnel and senior veterinary students under the supervision of veterinarians. A standard cautery disbudding protocol was used: the surrounding area was clipped, and a butane-fueled cautery dehorner (Portasol Calf Dehorner III, $15 \mathrm{~mm}$ tip; Portasol USA, Elmira, OR) was used to remove the horn bud germinal epithelium including the burned necrotic center of the bud. The contact time of the instrument to the epithelium was a maximum of $5 \mathrm{~s}$. For the dehorning procedure, operators restrained the animals by standing over the calves while facing forward to limit neck and body movement. All operators held the dehorner in the right hand for the right bud and the left hand for the left bud, independent of their dominant hand. Prior to disbudding, local anesthesia using $4 \mathrm{~mL}$ of $2 \%$ lidocaine (VetOne, MWI Animal Health, Boise, ID) mixed with $1 \mathrm{~mL}$ of $8.4 \%$ sodium bicarbonate (VetOne) was injected bilaterally as a cornual nerve block, and meloxicam [ZyGenerics (Zydus, Pennington, NJ) 15-mg tablets; $45 \mathrm{mg}$ per calf] was administered orally for postdisbudding analgesia.

Prior to disbudding, operators were blinded to treatment assignment. Treatment with ALU (AluSpray, NeogenVet, Lexington, KY) was randomly allocated to the right or left horn bud within each animal at the time of disbudding. After disbudding was completed, the operator was informed which horn bud received ALU treatment. A computer-created, random number list was created for each farm with blocks of 10 animals to control for weekly and inter-operator variability as well as inter-farm variability. The control bud did not receive any treatment, whereas the treatment bud received ALU. Treatment was applied directly to the disbudding site and up to $2 \mathrm{~cm}$ on the skin surrounding the wound. After applying the treatments, operators could not be blinded to treatment as the ALU product has a bright, shiny, silver coloration. Finally, $0.5 \%$ permethrin fly and maggot spray (Catron, Bayer, Shawnee Mission, KS) was applied topically on the surrounding skin of both horn buds to control for myiasis. Calves were then observed on a weekly basis over a 3 -wk follow-up period by a trained observer.

\section{Measured Outcomes}

A lesion score (LS) system for healing of the disbudding site was defined before the start of the experiment. Lesion score was defined as follows: LS1 $=$ no scab or discharge present (Figure 1A), LS2 = crusted and scab-filled wound or raised scabs present (Figure 1B), 
and LS3 = dried or moist purulent discharge (Figure 1C). The wound diameter (WD) measured across the inner edges of the cautery ring at the widest point of the disbudding site was used as a gauge of healing speed (Figure 1D and 1E). Lesion score and WD were determined by a trained observer on a weekly basis during the 3 -wk follow-up period. Initial WD immediately after disbudding were not measured to avoid contamination and to decrease the stress imposed to calves. Based on the diameter of the disbudding instrument, initial WD were considered to be $15 \mathrm{~mm}$. Due to the bright, shiny silver coloration of the ALU product and adherence to the wound site, it was not possible to blind the trained observer to treatment during the follow-up period. However, they were blind to the LS and WD data for the previous weeks because new data collection spreadsheets were generated for each week without the inclusion of the previous week's results.

\section{Statistical Analysis}

A sample size calculation was performed to find a minimum of a $50 \%$ reduction on LS $\geq 2$ in treated buds compared with nontreated buds. Based on the prevalence of LS $\geq 2$ observed in the farms before the beginning of the study, it was assumed that $40 \%$ of calves would have LS $\geq 2$ if no treatment was applied. Therefore, a minimum of 168 calves were necessary to detect the hypothesized reduction on LS $\geq 2$ with a $95 \%$ confidence interval and a power of $80 \%$.

All the descriptive and statistical analyses were performed using SAS version 9.4 (SAS Institute Inc., Cary, NC). Descriptive statistics were generated using FREQ and UNIVARIATE procedures. Pearson chi-squared test was performed to correct for multiple comparisons between the proportions of LS in treatment groups across the weeks during the follow-up period. Comparisons between the proportions of disbudding sites with different LS among treatment groups within weeks postdisbudding were obtained using the GENMOD procedure.

Disbudding site LS was further dichotomized to facilitate statistical analysis and data interpretation. Disbudding sites with LS $=1$ were considered to have normal healing (NH), whereas disbudding sites with LS $\geq 2$ were considered to have delayed healing (DH). Pearson correlation was used to compare DH during the first 3 wk postdisbudding, using the CORR procedure with the option Pearson for each week postdisbudding individually.

Logistic regression models were used to determine the odds of DH during the 3 wk postdisbudding. Initially, a logistic regression analysis was used to determine the effect of treatment on disbudding site healing during

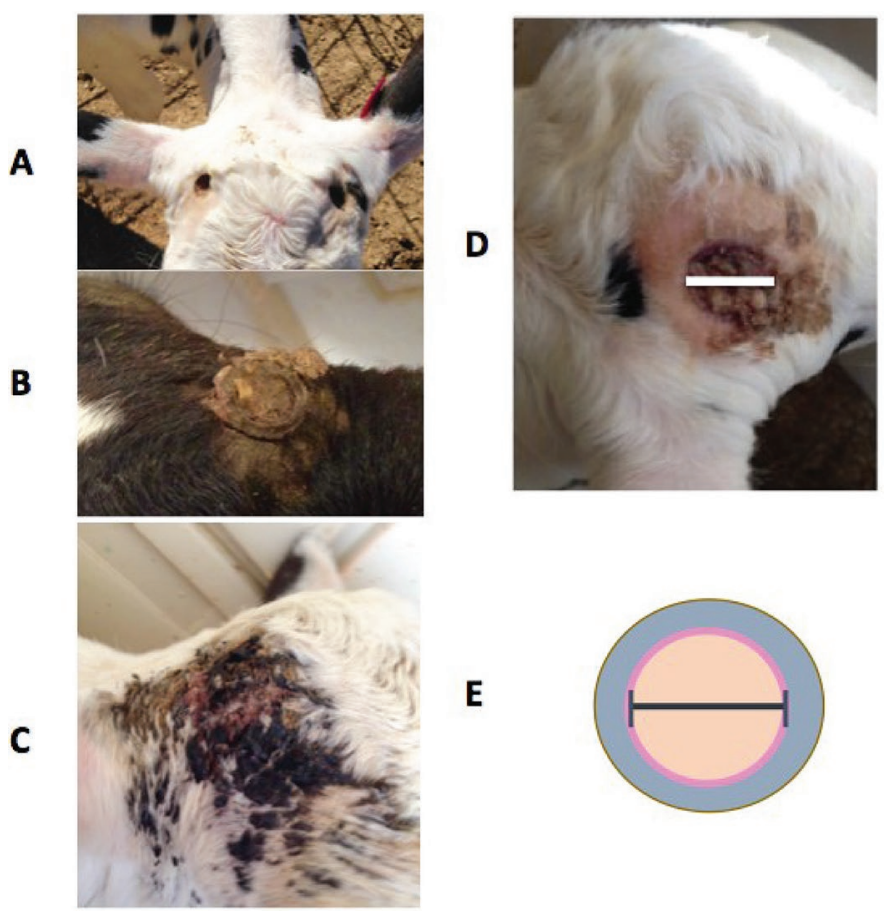

Figure 1. Disbudding lesion score (LS; 1, 2, or 3) and wound diameter (WD) were observed at 1,2 , and 3 wk postdisbudding. (A) LS1 was defined as no scabbing or discharge present, (B) LS2 was defined as scabs or raised crusts, and (C) LS3 was defined as having moist or dried purulent discharge. Halo diameter measurements (D-E) were obtained from the ring left over where the horn bud used to be. The diameter was measured in millimeters across from the inner edges of the ring at the widest point. Color version available online.

the 3 wk postdisbudding independent of other factors. Afterward, logistic regression models were used to determine the effect of the independent variables [i.e., treatment group, age in days when disbudding was performed, dehorning operator, blood total protein in wk 1 of life, and DH in wk 1 or 2 (or both) postdisbudding] and interactions on the dependent variables: healing at wk 2 and 3 postdisbudding. Treatment groups and other potential explanatory variables were forced into the models, and interactions were removed from the logistic models in a manual backward stepwise fashion if $P>0.1$. The Hosmer-Lemeshow goodness-of-fitness test was used as a diagnostic test to confirm that the models used did not violate the assumptions of logistic regression.

The progress of WD $(\mathrm{mm})$ during the 3 wk postdisbudding for the treatment groups accounting for the explanatory variables was analyzed by MIXED procedure in SAS, with repeated statement and Bonferroni adjustment for multiple comparisons. A Kenward-Roger degrees of freedom approximation was used to calculate the denominator degrees of freedom and the covariance structure used for each model was chosen based on the 


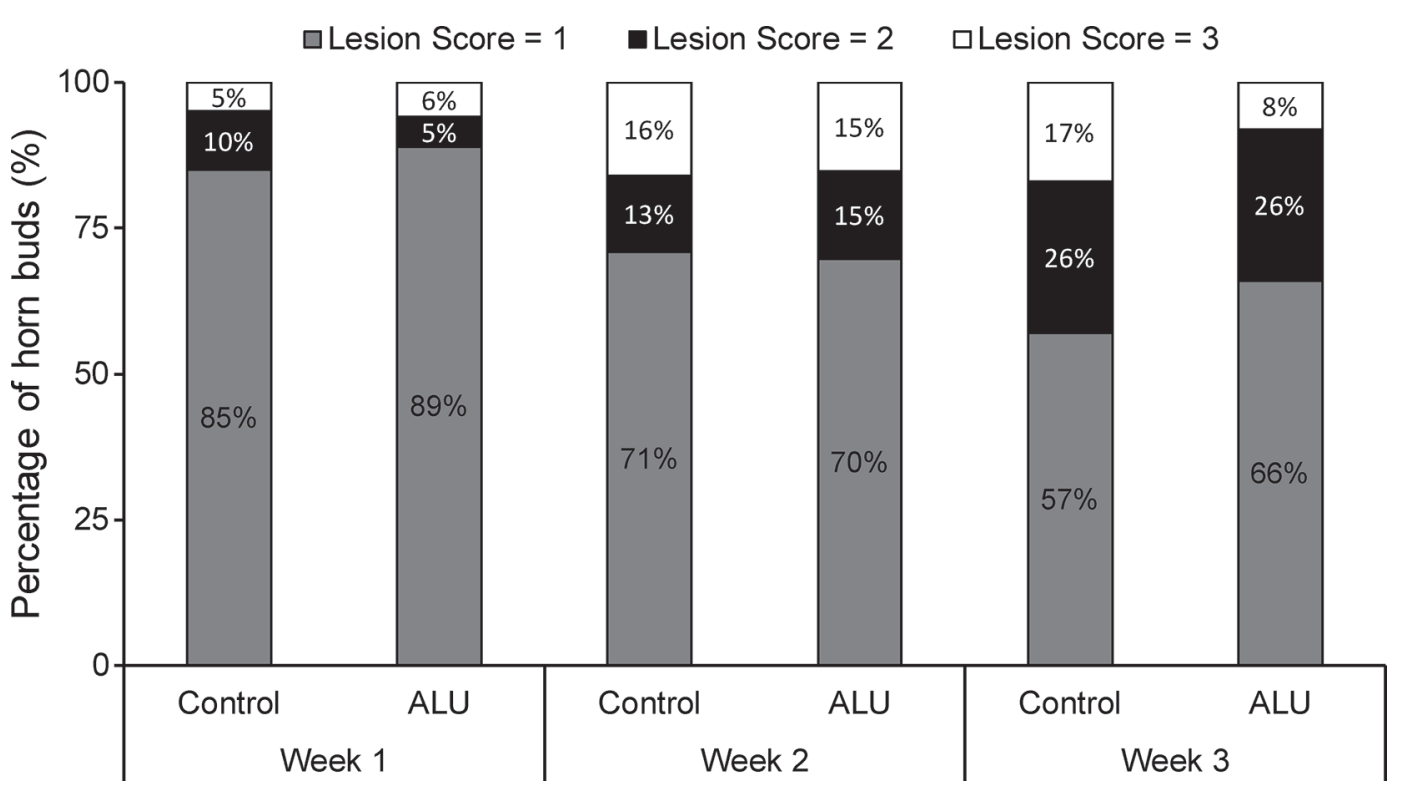

Figure 2. Proportion of animals at wk 1 to 3 with different lesion scores (LS) by treatment groups: control and aluminum bandage spray $(\mathrm{ALU}) . \mathrm{LS} 1=$ no scab or discharge present, LS2 $=$ crusted and scab-filled wound or raised scabs present, and LS3 $=$ dried or moist purulent discharge.

smallest Akaike information criterion. Farm was used as a random effect for statistical analysis models, and results were considered statistically significant when $P$ $\leq 0.05$ and tendency was considered when $0.05<P \leq$ 0.10 .

\section{RESULTS}

\section{Descriptive Results}

A total of 220 animals were enrolled in the study; however, 11 animals were lost to follow-up due to mortality for reasons unrelated to the study. All animals enrolled in the study were still alive for the first measurement postdisbudding, thereafter 7 animals were lost between wk 1 and 2 measurements and another 4 animals were lost before wk 3 postdisbudding visit. The exact cause for these losses was undocumented, but the losses were unrelated to the disbudding procedure.

The proportion of calves with different LS for both groups across wk 1 through 3 is shown in Figure 2. The proportion of calves with LS $=1$ across the all 3 wk postdisbudding for both treatment groups was not different $(P=0.66)$, whereas calves with $\mathrm{LS}=2(P$ $=0.10)$ and $\mathrm{LS}=3(P=0.06)$ tended to be different across the follow-up period. When analyzing the difference in LS proportion among treatment groups during wk 1 postdisbudding, the proportion of LS tended to differ in wk $1(P=0.06)$, whereas no difference was observed in wk $2(P=0.91)$ and a significant differ- ence was observed in wk $3(P=0.02)$. Interestingly, we observed that the proportion of disbudding sites with $\mathrm{LS}=2$ during wk 2 , and $\mathrm{LS}=3$ on wk 3 were about 2 times higher in the control group when compared with ALU: 10 versus $5 \%$ for control and ALU, respectively, in wk 2, and 17 versus $8 \%$ for control and ALU, respectively, in wk 3.

\section{Correlations of Week and Treatment on Delayed Healing}

The correlation between weeks postdisbudding and $\mathrm{DH}$, by treatment group, is presented in Table 1. Presence of DH in wk 2 postdisbudding was positively correlated with DH during wk 1 for both groups. Similarly, DH in wk 3 was positively correlated with $\mathrm{DH}$ in wk 1 , 2 , or 1 and $2(P=0.04, P<0.001, P=0.003$; respectively) for the control group. However, DH in wk 3 was only correlated significantly with $\mathrm{DH}$ in wk 2 for the ALU group $(P<0.001)$; with only a tendency observed when analyzing the correlation between wk 1 or 1 and 2 combined $(P=0.10)$.

\section{Odds of Delayed Healing Postdisbudding}

The odds of DH during a 3-wk follow-up period independent of the healing status of the previous weeks are presented in Table 2. Without considering $\mathrm{DH}$ in the previous week, there was no effect of treatment in wk 1 and $2(P=0.2$ and $P=0.8$, respectively $)$. However, 
Table 1. Correlation between delayed healing for the different followup weeks postdisbudding according to treatment groups

\begin{tabular}{lccccc}
\hline & \multicolumn{2}{c}{ Wk 2 } & & \multicolumn{2}{c}{ Wk 3} \\
\cline { 2 - 3 } \cline { 5 - 6 } Item & $\mathrm{R}^{2}$ & $P$-value & & $\mathrm{R}^{2}$ & $P$-value \\
\hline Control $^{1}$ & & & & & \\
Wk 1 & 0.25 & $<0.001$ & & 0.14 & 0.04 \\
Wk 2 & & & 0.38 & $<0.001$ \\
Wk 1 and 2 & & & & 0.20 & 0.003 \\
ALU & & & & 0.11 & 0.10 \\
Wk 1 & 0.36 & $<0.001$ & & 0.25 & $<0.001$ \\
Wk 2 & & & & 0.11 & 0.10 \\
Wk 1 and 2 & & & & \\
\hline
\end{tabular}

${ }^{1}$ Control disbudding sites were not treated with aluminum bandage (ALU) after disbudding.

${ }^{2} \mathrm{Wk} 1$ and 2 were defined as disbudding sites with delayed healing during the first 2 wk after disbudding.

${ }^{3} \mathrm{ALU}$ disbudding sites were treated with aluminum bandage spray after disbudding.

the control group was 1.44 times more likely to have DH when compared with the ALU group in wk $3(P$ $=0.07$ ).

Further statistical analyses were performed to determine the influence of LS in the previous weeks and DH at a given week during the follow-up period with results presented in Table 3. Briefly, disbudding wound sites with $\mathrm{DH}$ in wk 1 postdisbudding were 6.05 times more likely to have $\mathrm{DH}$ in wk 2 with no effect of treatment $(P$ $<0.001$ ). Controlling for DH in the wk 2, treatment had an effect on DH in wk 3 with control animals 1.53 times more likely to have DH compared with ALU calves ( $P$ $=0.05$ ). Controlling for treatment, $\mathrm{DH}$ in wk 3 was 3.23 times more likely to occur if $\mathrm{DH}$ occurred during wk 1 and $2(P=0.002)$; when controlling for $\mathrm{DH}$, the control group tended $(P=0.07)$ to be 1.45 times more likely to have $\mathrm{DH}$ when compared with ALU.

\section{Effect of Week and Treatment on Wound Diameter}

The dynamics of WD during the first 3 wk postdisbudding for both study groups are presented in Figure 3. A treatment by time interaction on WD was observed $(P=0.04)$. Wound diameter was about $1 \mathrm{~mm}$ larger on both treatment groups at wk $1(\mathrm{WD}=16.1$ $\pm 0.18 \mathrm{~mm}$ for $\mathrm{ALU}$, and $\mathrm{WD}=15.9 \pm 0.18 \mathrm{~mm}$ for control; $P=0.99$ ) when compared with the $15 \mathrm{~mm}$ baseline. For the control disbudding sites, WD continued to increase during wk $2(\mathrm{WD}=16.3 \pm 0.17 \mathrm{~mm})$ and then decreased in wk 3 (WD $=15.4 \pm 0.24 \mathrm{~mm}$, $P=0.1$ ), whereas for the treatment group, WD did not change in wk $2(\mathrm{WD}=16.1 \pm 0.17 \mathrm{~mm})$ and was more than $1 \mathrm{~mm}$ smaller in wk $3(\mathrm{WD}=14.7 \pm 0.24$ $\mathrm{mm}, P \leq 0.001$ ) when compared with the wk 1 measurement. Wound diameter was significantly smaller in treated versus control calves at wk 3 postdisbudding $(P$ $=0.04)$. Additionally, WD only decreased below initial diameter of the disbudding site after 3 wk for ALU; control disbudding sites had diameters larger than 15 $\mathrm{mm}$ for the duration of the study period.

\section{DISCUSSION}

The goal of this study was to assess wound healing following cautery disbudding in dairy calves with and without the use of a topical aluminum spray. Our results show a high prevalence of $\mathrm{DH}$ within the 3 -wk postdisbudding follow-up period. Furthermore, calves that developed DH were more likely to continue to have abnormal healing into the subsequent week. Differences between the control and ALU groups were detected in wk 3 postdisbudding for both LS and WD data, indicating that ALU resulted in improved healing by wk 3 .

Several explanations are possible for the observation that the effects of ALU on improving LS and WD were

Table 2. Logistic regression of the association of aluminum bandage (ALU) treatment after disbudding and delayed healing during the weeks postdisbudding without considering the occurrence of lesion score $\geq 2$ in the previous week $(\mathrm{s})$

\begin{tabular}{|c|c|c|c|c|}
\hline Item & $\begin{array}{c}\text { Prevalence of } \\
\text { delayed healing }(\%)\end{array}$ & Odds ratio & $95 \%$ CI & $P$-value \\
\hline \multicolumn{5}{|c|}{ Wk 1 postdisbudding } \\
\hline $\mathrm{ALU}^{1}$ & 10.5 & Referent & - & - \\
\hline Control $^{2}$ & 14.4 & 1.42 & $0.79-2.56$ & 0.2 \\
\hline \multicolumn{5}{|c|}{ Wk 2 postdisbudding } \\
\hline ALU & 29.7 & Referent & - & - \\
\hline Control & 28.7 & 0.96 & $0.62-1.46$ & 0.8 \\
\hline \multicolumn{5}{|c|}{ Wk 3 postdisbudding } \\
\hline ALU & 34.4 & Referent & - & - \\
\hline Control & 43.1 & 1.44 & $0.97-2.14$ & 0.07 \\
\hline
\end{tabular}




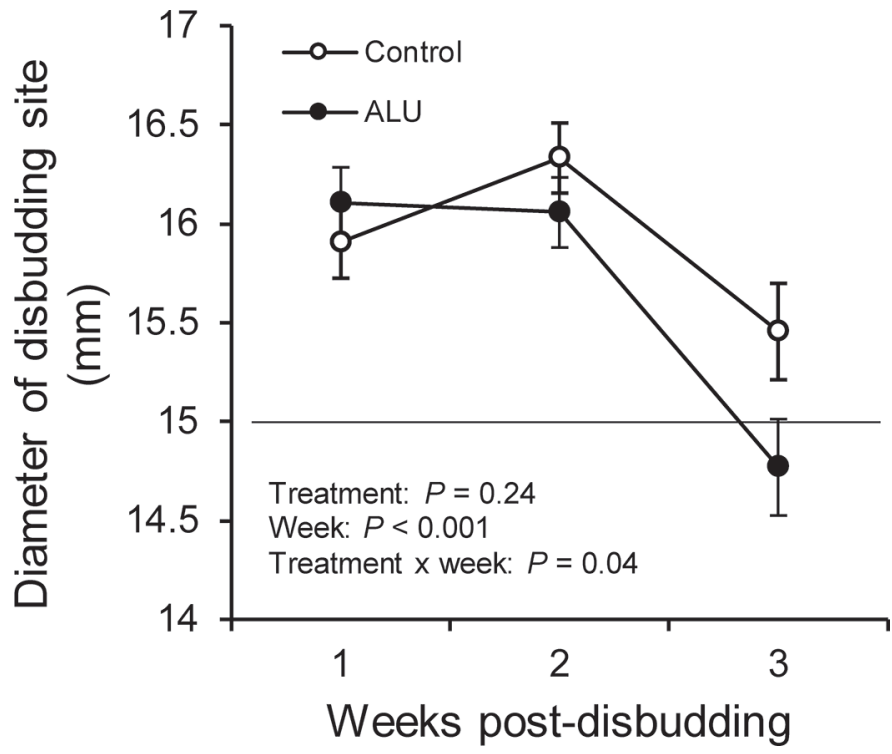

Figure 3. Wound diameter dynamics during the first 3 wk postdisbudding according to treatment groups. The horizontal line at 15 $\mathrm{mm}$ indicates the initial diameter of the disbudding site immediately after disbudding. ALU = aluminum bandage spray. Each time point represents LSM \pm SEM for all the animals examined at a given week.

not detected until wk 3. We can speculate that ALU may be playing a role in increasing the rate of healing, or conversion to a $\mathrm{LS}=1$, for wounds undergoing in- flammation following thermal burns caused by cautery disbudding. One reason this difference was not detected in wk 1 or 2 may be that the postdisbudding wounds are undergoing a process of inflammation as part of the natural healing process that our simple LS system was unable to differentiate. Thus, we may not have been able to capture the healing effects of ALU in those first 2 wk using a 3-point LS characterization. If we had measured LS and WD for a longer time period, into wk 4 and 5, or more frequently, the effects of ALU on healing may have become more evident. Another potential factor that might account for the pattern of healing observed in this study was the use of permethrin fly spray surrounding the wound sites, which may have enhanced or diminished the effects of ALU. Because permethrin was used on both disbudding wounds, the effects of ALU compared with controls were not independently evaluated from the use of this product.

The ALU label claims that the product works as a waterproof barrier protectant, does not damage wound tissues, adheres to the skin, allows the skin to oxygenate, and acts as a dry barrier against mud, debris, and sensitivity to sunlight. The exact mechanism of action for ALU is currently unknown, and the product may be acting at one of several stages in the healing of thermal wounds. In superficial burn wounds, the stages of wound healing include hemostasis, inflamma-

Table 3. Logistic regression of the association of aluminum bandage (ALU) treatment after disbudding and delayed healing during the weeks postdisbudding considering the occurrence of delayed healing in the previous week $(\mathrm{s})$

\begin{tabular}{|c|c|c|c|c|}
\hline Item & $\begin{array}{c}\text { Prevalence of } \\
\text { delayed healing (\%) }\end{array}$ & Odds ratio & $95 \% \mathrm{CI}$ & $P$-value \\
\hline \multicolumn{5}{|c|}{ Wk 2 postdisbudding } \\
\hline \multicolumn{5}{|c|}{ Treatment } \\
\hline $\mathrm{ALU}^{1}$ & 10.5 & Referent & - & - \\
\hline Control $^{2}$ & 14.4 & 0.87 & $0.56-1.36$ & 0.5 \\
\hline \multicolumn{5}{|c|}{ Wk 1 healing } \\
\hline $\mathrm{NH}^{3}$ & 87.6 & Referent & - & - \\
\hline $\mathrm{DH}^{4}$ & 12.4 & 6.05 & $3.25-11.26$ & $<0.001$ \\
\hline \multicolumn{5}{|c|}{ Wk 3 postdisbudding } \\
\hline \multicolumn{5}{|c|}{ Treatment } \\
\hline ALU & 29.7 & Referent & - & - \\
\hline Control & 28.7 & 1.53 & $1.00-2.32$ & 0.05 \\
\hline \multicolumn{5}{|c|}{ Wk 2 healing } \\
\hline NH & 70.8 & Referent & - & - \\
\hline $\mathrm{DH}$ & 29.2 & 4.33 & $2.77-6.79$ & $<0.001$ \\
\hline \multicolumn{5}{|c|}{ Wk 3 postdisbudding } \\
\hline \multicolumn{5}{|c|}{ Treatment } \\
\hline ALU & 34.4 & Referent & - & - \\
\hline Control & 43.1 & 1.45 & $0.97-2.17$ & 0.07 \\
\hline \multicolumn{5}{|c|}{ Wk 1 and 2 healing } \\
\hline NH & 91.9 & Referent & - & - \\
\hline DH & 8.1 & 3.23 & $1.55-6.74$ & 0.002 \\
\hline
\end{tabular}

${ }^{1} \mathrm{ALU}$ disbudding sites were treated with aluminum bandage spray after disbudding.

${ }^{2}$ Control disbudding sites were not treated with aluminum bandage spray after disbudding.

${ }^{3} \mathrm{NH}=$ normal healing, defined as disbudding site with no scabbing or discharge present in a given week.

${ }^{4} \mathrm{DH}=$ delayed healing, defined as disbudding site presenting scabs or discharge (or both) in a given week. 
tion, proliferation, and maturation and remodeling of the tissues, including wound contraction, granulation, and re-epithelialization. The difference between burn wounds and other traumatic wounds is that they are characterized by fundamental damage to vital tissues, which complicates the normal wound healing response, as cells and vasculature are often destroyed, leading to a region of coagulative necrosis (Rose and Chan, 2016). The ALU may be acting as a drying or astringent agent to accelerate wound contracture or reduce inflammation/infection associated with coagulative necrosis. It is not known how ALU would affect healing of other types of wounds.

Alternative topical treatments for burn wounds in humans and in animals are limited with little evidence for efficacy. Studies on other topical products, such as silver sulfadiazine, show that this product has mixed evidence for efficacy in various species (Miller et al., 2012); nevertheless, the use or storage of silver sulfadiazine is not permitted in grade "A" dairy operations in the United States. Zinc-related products have previously been evaluated in porcine (Agren et al., 1991) and rat (Otsuka et al., 2015) models with evidence that initial inflammation in superficial skin wounds or thermal burns can be controlled, and re-epithelialization can be enhanced with these products. With regard to the aluminum-based aerosol spray product used in this study, no data have been published on the mechanism of action of aluminum powder with respect to wound healing; therefore, future work is required to determine the exact mechanism of action by which the product enhances wound healing.

Because culture or histopathology was not performed in the current study, it is unknown if the observed scabs or purulent discharge represent an inflammatory process associated with a thermal burn or a secondary bacterial infection. In either case, inflammation associated with the immediate postdisbudding period has been shown to result in continued cortisol and behavioral changes linked to pain (McMeekan et al., 1998; Stafford and Mellor, 2005). Therefore, it is possible that chronic inflammation due to a natural process or infection could be a constant noxious stimulus to affected calves. The pain response associated with prolonged healing and infection or inflammation of disbudding wounds in the absence or presence of analgesia have not been previously measured. Methods such as pressure algometry and nociceptive thresholds may be used in the future to better understand the relationship between disbudding, ALU, and pain.

The current study did not measure the effect of delayed wound healing on calf production parameters, such as milk intake or weight gain with respect to ALU. Bates et al. (2015) assessed the effect of analgesic protocols at disbudding on average daily weight gain and milk intake of dairy calves, and found evidence that dairy calves disbudded with analgesia were more productive. However, in that study there was an interaction between efficacy of the analgesic protocol on production parameters and operator skill level (farmer compared with veterinarian), and time frame relative to disbudding that the protocol had an effect. In the present study, operators of various skill level (veterinarians and veterinary students) performed the procedure, which is one limitation of the study. The authors acknowledge this limitation but designed the study in a randomized block design to account for this variation in skill level.

Understanding the health effects of DH postdisbudding on calf growth and long-term health would be useful to the dairy industry to guide management decisions and disbudding protocols. Additionally, the use of polled sires when breeding dairy cattle should be considered as a more long-term solution to the pain and healing complications of disbudding and dehorning in cattle. Because the polled trait results from an autosomal dominant pattern of inheritance, genetic selection for polled cattle could reduce the prevalence of the horned trait (Spurlock et al., 2014). Although polled dairy sires are commercially available, the use of polled genetics is not common, where $94 \%$ of US dairies report disbudding or dehorning their animals (USDA, 2009). Until polled sires are commonly used throughout the industry, disbudding procedures will remain a standard aspect of dairy calf processing. Therefore, mitigating disbudding wound complications through a better understanding of the healing process and wound management should be considered to improve calf growth and welfare.

\section{CONCLUSIONS}

This study found that a topical aluminum bandage spray improves wound healing during the $3 \mathrm{wk}$ following disbudding. Dairy producers and veterinarians who routinely disbud their dairy calves should consider using topical aluminum barrier spray to improve wound healing. Though the pain response postdisbudding has been studied extensively to improve animal welfare on dairy farms, previous studies have not directly measured wound healing postdisbudding as a model for the pain response and animal welfare following disbudding procedures. One reason for this might be because delayed wound healing may only become apparent as long-term effects, and previously measured indices of pain may not be a good proxy to capture differences in healing as it relates to pain and production measures in dairy calves. This study highlights the need to under- 
stand wound healing postdisbudding, and the need for novel management interventions to ameliorate delayed wound healing in dairy calves using topical treatments.

\section{ACKNOWLEDGMENTS}

None of the authors has any financial or personal relationships with other people or organization that could inappropriately influence or bias the content of the paper. This research was funded by the Colorado State University Dairy Field Service part of the Veterinary Teaching Hospital. The authors thank the farm owners and staff for the use of their animals, facilities, and equipment during this project. We also thank the veterinary students of the Colorado State University, College of Veterinary Medicine and Biomedical Sciences for their assistance with animal handling and data collection.

\section{REFERENCES}

Agren, M. S., M. Chvapil, and L. Franzen. 1991. Enhancement of re-epithelialization with topical zinc oxide in porcine partial-thickness wounds. J. Surg. Res. 50:101-105.

Aubry, P. 2005. Routine surgical procedures in dairy cattle under field conditions: Abomasal surgery, dehorning, and tail docking. Vet. Clin. North Am. Food Anim. Pract. 21:55-72.

Bates, A. J., P. Eder, and R. A. Laven. 2015. Effect of analgesia and anti-inflammatory treatment on weight gain and milk intake of dairy calves after disbudding. N. Z. Vet. J. 63:153-157.

Kihurani, D. O., S. M. Mbiuki, and T. A. Ngatia. 1989. Healing of dehorning wounds. Br. Vet. J. 145:580-585.

Loxton, I. D., M. A. Toleman, and A. E. Holmes. 1982. The effect of dehorning Brahman crossbred animals of four age groups on subsequent bodyweight gain. Aust. Vet. J. 58:191-193.
Marshall, B. L. 1977. Bruising in cattle presented for slaughter. N. Z. Vet. J. 25:83-86.

McMeekan, C. M., K. J. Stafford, D. J. Mellor, R. A. Bruce, R. N. Ward, and N. G. Gregory. 1998. Effects of regional analgesia and/ or a non-steroidal anti-inflammatory analgesic on the acute cortisol response to dehorning in calves. Res. Vet. Sci. 64:147-150.

Miller, A. C., R. M. Rashid, L. Falzon, E. M. Elamin, and S. Zehtabchi. 2012. Silver sulfadiazine for the treatment of partial-thickness burns and venous stasis ulcers. J. Am. Acad. Dermatol. 66:e159e165.

Neely, C. D., D. U. Thomson, C. A. Kerr, and C. D. Reinhardt. 2014. Effects of three dehorning techniques on behavior and wound healing in feedlot cattle. J. Anim. Sci. 92:2225-2229.

Otsuka, M., H. Hatakeyama, M. Shikamura, K. Otsuka, and A. Ito. 2015. Therapeutic effects of transdermal systems containing zincrelated materials on thermal burn rats. Biomed. Mat. Eng. 25:143156

Ramsay, W. R., H. R. Meischke, and B. Anderson. 1976. The effect of tipping of horns and interruption of journey on bruising in cattle. Aust. Vet. J. 52:285-286.

Robbins, J., D. Weary, C. Schuppli, and M. Von Keyserlingk. 2015. Stakeholder views on treating pain due to dehorning dairy calves. Anim. Welf. 24:399-406.

Rose, L. F., and R. K. Chan. 2016. The burn wound microenvironment. Adv. Wound Care (New Rochelle) 5:106-118.

Spurlock, D. M., M. L. Stock, and J. F. Coetzee. 2014. The impact of 3 strategies for incorporating polled genetics into a dairy cattle breeding program on the overall herd genetic merit. J. Dairy Sci. 97:5265-5274.

Stafford, K. J., and D. J. Mellor. 2005. Dehorning and disbudding distress and its alleviation in calves. Vet. J. (London, England) 169:337-349

Stock, M. L., S. L. Baldridge, D. Griffin, and J. F. Coetzee. 2013 Bovine dehorning: Assessing pain and providing analgesic management. Vet. Clin. North Am. Food Anim. Pract. 29:103-133.

USDA. 2009. Dairy 2007, Part V: Changes in dairy cattle health and management practices in the United States, 1996-2007. \#519.0709. USDA:APHIS:VS, CEAH, Fort Collins, CO. 